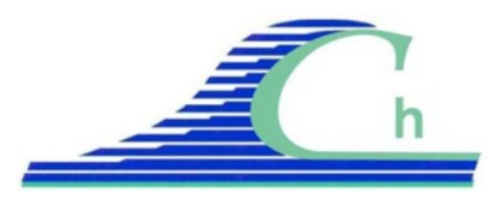

XII èmes Journées Nationales Génie Côtier - Génie Civil

Cherbourg, 12-14 juin 2012

DOI:10.5150/jngcgc.2012.099-B C Editions Paralia CFL

disponible en ligne - http://www.paralia.fr-available online

\title{
Contributions des vagues dans la surcote associée à la tempête Xynthia, février 2010
}

\author{
Xavier BERTIN ${ }^{1}$, Kai LI ${ }^{1}$, Aron ROLAND ${ }^{2}$, \\ Jean-François BREILH ${ }^{1}$, Eric CHAUMILLON ${ }^{1}$
}

\section{UMR 7266 LIENSs CNRS-Université de La Rochelle,}

Institut du Littoral et de l'Environnement, 2 rue Olympe de Gouges,

17000 La Rochelle, France.

xbertin@univ-lr.fr

2. Institute for Hydraulic Engineering and Water Resources Management.

Technical University of Darmstadt, Germany.

aaronroland@gmx.de

\section{Résumé :}

La tempête Xynthia a sévèrement touché les littoraux situés dans la partie centrale du Golfe de Gascogne dans la nuit du 27 au 28 février 2010, en générant une surcote de plus d'1.50 m au marégraphe de La Pallice (La Rochelle). Cette surcote était en phase avec une marée haute de vives-eaux, ce qui a entrainé la submersion de nombreux cordons dunaires et inondé les zones basses du littoral. Notre étude vise à étudier les contributions des vagues dans la surcote associée à Xynthia, en combinant modélisation numérique et mesures in situ. Un nouveau système de modélisation numérique, basé sur le code de circulation océanique SELFE et le modèle spectral de vagues WWMII, a été mis en place pour l'Atlantique nord-est. Le couplage entre les vagues et les courants est complet en $2 \mathrm{DH}$ et en $3 \mathrm{D}$ et les deux modèles partagent la même grille de calcul non structurée et la même décomposition de domaine. Une fois calibré, ce système de modélisation numérique permet de reproduire les niveaux d'eau pendant Xynthia avec une erreur de l'ordre de $0.10 \mathrm{~m}$ et les hauteurs et périodes des vagues avec des erreurs de l'ordre de 15\%. L'analyse des résultats numériques montre d'abord que la surcote a été amplifiée par la présence de vagues très jeunes et cambrées, qui augmentent la rugosité de l'océan. Cet état de mer est lié à la trajectoire atypique de la tempête Xynthia, qui a traversé le Golfe de Gascogne du SO vers le NE, réduisant la zone de génération des vagues à quelques centaines de kilomètres. A la côte, les gradients de tension de radiation des vagues contribuent à la surcote totale de quelques centimètres dans les ports à plus de $0.40 \mathrm{~m}$ au niveau des plages directement exposées aux houles océaniques. Cette étude montre que, pendant la tempête Xynthia, les vagues ont autant contribué à la surcote que le vent et les gradients de pression atmosphériques.

\section{Mots-clés :}

Surcote - Xynthia - Vagues - Pertuis charentais 


\section{Introduction}

Du fait de la présence d'un plateau continental large et de leur position sur la trajectoire des tempêtes hivernales, les littoraux d'Europe du nord-ouest sont soumis à des surcotes qui dépassent régulièrement le mètre. Les côtes basses, comme celles de la mer de Wadden ou des Pertuis Charentais, sont donc vulnérables à la submersion marine. La tempête Xynthia, qui a touché durement les littoraux de Vendée et de Charente Maritime dans la nuit du 27 au 28 février 2010, est venue rappeler tragiquement cette vulnérabilité. Une dépression à $970 \mathrm{hPa}$ accompagnée de vents de $\mathrm{SO}$ soufflant de 100 à $130 \mathrm{~km} / \mathrm{h}$ (jusqu'à $160 \mathrm{~km} / \mathrm{h}$ sur l'île de Ré) a généré une surcote de plus d'1.50 m au marégraphe de La Pallice (La Rochelle). Cette surcote a coïncidé avec une marée haute de vives-eaux, si bien que le niveau de la mer a atteint la cote record de $4.5 \mathrm{~m}$ NGF à La Pallice. De nombreux cordons dunaires et digues ont été submergés, inondant de larges zones et occasionnant la mort de 47 personnes en Charente-Maritime et Vendée ainsi que des dégâts matériels colossaux. Le cas de la tempête Xynthia est très intéressant sachant que des travaux récents suggèrent que la période de retour d'une telle surcote soit supérieure à 50 ans (BERNARDARA et al., 2011) alors que deux autres tempêtes d'intensité équivalente ont récemment frappé ce secteur (tempêtes Martin et Klaus en 2009). Notre équipe a déjà réalisé une simulation rétrospective de la tempête Xynthia à l'échelle du Golfe de Gascogne et a montré l'importance de l'âge des vagues et de la résonance de l'onde de tempête sur le plateau continental (BERTIN et al., 2012). L'objectif de cette étude est d'analyser avec une résolution beaucoup plus fine (i.e. représentation des zones de déferlement) la contribution des processus induits par les vagues dans la surcote associée à Xynthia.

\section{Zone d'étude}

Notre étude se focalise sur la partie centrale du Golfe de Gascogne, caractérisée par un plateau continental large (figure 1). Le régime de marée est méso- à macro-tidal (marnage de vives-eaux $>6 \mathrm{~m}$ dans les Pertuis Charentais) et semi-diurne. Le régime d'agitation dans le Golfe de Gascogne a été analysé par DUPUIS et al. (2006), qui montrent que les hauteurs significatives (Hs) moyennes annuelles varient de 1.36 à Biscarosse à 1.81 à Yeu (figure 1) alors que Hs maximales dépassent $9 \mathrm{~m}$ pour ces deux sites. Les périodes moyennes annuelles sont de l'ordre de 6 à 7 secondes alors que les périodes de pic peuvent occasionnellement dépasser $20 \mathrm{~s}$. Sur la base d'une simulation numérique rétrospective sur la période 1953-2009, DODET et al. (2010) ont montré que les directions moyennes (Dir) annuelles variaient de $300^{\circ} \mathrm{N}$ à $270^{\circ} \mathrm{N}$ du sud au nord de la zone. 


\section{XII ${ }^{\text {èmes }}$ Journées Nationales Génie Côtier - Génie Civil \\ Cherbourg, 12-14 juin 2012}

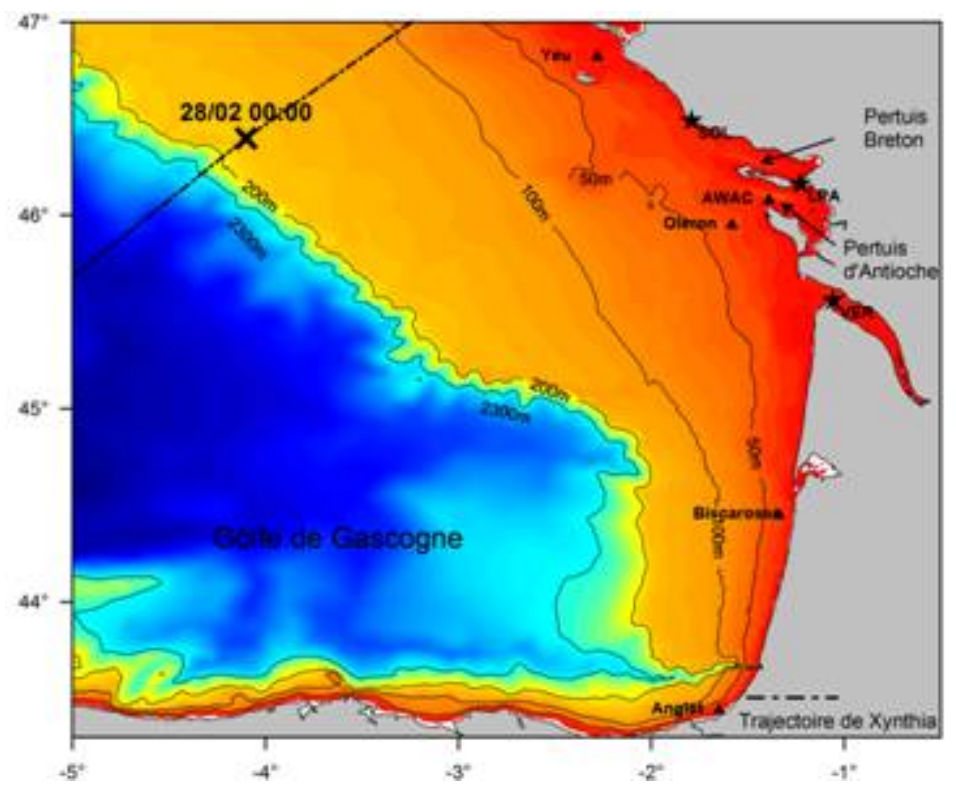

Figure 1. Bathymétrie du Golfe de Gascogne, trajectoire du centre de la tempête Xynthia, marégraphes (étoiles : Les Sables d'Olonnes, La Pallice et Verdon) et houlographes (triangles) utilisés pour la validation du modèle.

\section{Le système de modélisation numérique}

\subsection{Description générale}

Notre étude repose sur le développement et l'application d'un nouveau système de modélisation numérique, basé sur le couplage entre le code de circulation hydrodynamique SELFE (ZHANG \& BAPTISTA, 2008) et le modèle spectral de vagues WWMII (ROLAND et al., 2009). Le couplage entre les vagues et les courants est complet en $2 \mathrm{DH}$ et $3 \mathrm{D}$ et les deux modèles partagent les mêmes grille de calcul non structurée et décomposition de domaine, ce qui rend ce système de modélisation très efficace d'un point de vue computationnel et autorise le calcul parallèle massif.

\subsection{Modèle de vagues}

Le code spectral de vagues WWMII (ROLAND et al., 2009) résout l'équation de conservation de la densité spectro-angulaire d'action des vagues. L'advection dans l'espace géographique est résolue sur la grille non-structurée, grâce à une méthode numérique de type Fluctuation Splitting Schemes (ABGRALL, 2006). WWMII calcule des paramètres moyens à partir du spectre d'énergie (tensions de radiation des vagues, vitesse de frottement, etc.) et échange ces variables avec SELFE directement par mémoire vive. Le modèle est forcé par des champs de vent provenant d'un assemblage entre des sorties du modèle GFS (Global Forecast System, NOAA) pour l'ensemble du domaine et du modèle ALADIN (Météo France) pour le Golfe de Gascogne et la manche (BERTIN et al., 2012). 


\subsection{Modèle de circulation hydrodynamique}

Le modèle de circulation hydrodynamique SELFE (ZHANG \& BAPTISTA, 2008) résout les équations de Navier-Stokes sur une grille triangulaire non-structurée. Dans le cadre de cette étude, nous utilisons SELFE en mode 2DH barotrope et les équations résolues sont donc les suivantes :

$$
\begin{aligned}
& \frac{\partial \zeta}{\partial t}+\vec{\nabla} \cdot \int_{-h}^{\zeta} \vec{u} d z=0 \\
& \frac{D U}{D t}=-f U+\alpha g \frac{\partial \varphi}{\partial x}-\frac{1}{\rho} \frac{\partial P_{A}}{\partial x}-g \frac{\partial \zeta}{\partial x}+\frac{\vec{\tau}_{S x}-\vec{\tau}_{B x}}{\rho(\zeta+h)}-\frac{1}{\rho(\zeta+h)} \cdot\left(\frac{\partial S_{x x}}{\partial x}+\frac{\partial S_{x y}}{\partial y}\right) \\
& \frac{D V}{D t}=f V+\alpha g \frac{\partial \varphi}{\partial y}-\frac{1}{\rho} \frac{\partial P_{A}}{\partial y}-g \frac{\partial \zeta}{\partial y}+\frac{\vec{\tau}_{S y}-\vec{\tau}_{B y}}{\rho(\zeta+h)}-\frac{1}{\rho(\zeta+h)} \cdot\left(\frac{\partial S_{y y}}{\partial y}+\frac{\partial S_{x y}}{\partial x}\right)
\end{aligned}
$$

Dans ces équations, $\zeta$ désigne l'élévation de la surface libre, $U$ et $V$ les composantes selon $x$ et $y$ de la vitesse intégrée sur la verticale, $g$ est l'accélération de la gravité, $f$ est la force de Coriolis, $\varphi$ est le potentiel de marée astronomique, $h$ est la bathymétrie, $S_{x x}$, $S_{x y}$ et $S_{y y}$ sont les tensions de radiation des vagues et $\tau_{b}$ est la contrainte de frottement au fond. $\tau_{s}$ correspond à la contrainte de surface et peut être calculée avec une paramétrisation de type quadratique ou dépendant des vagues. L'équation de continuité est résolue par une méthode de volumes finis alors que les équations de conservation de la quantité de mouvement sont résolues par une méthode de Galerkin continue. Le traitement Lagrangien des termes advectifs garantit une excellente stabilité numérique, même avec de grands pas de temps hydrodynamiques. SELFE est forcé le long de sa frontière ouverte par le modèle régional de marée de PAIRAUD et al. (2006) et par le même forçage atmosphérique que celui utilisé par WWMII.

\subsection{Implémentation du système de modélisation}

L'espace géographique est discrétisé par une grille constituée d'éléments triangulaires et dont l'extension couvre l'Océan Atlantique Nord-Est (figure 2). Cette grille possède 502798 éléments (260622 nœuds), dont la taille varie de $30 \mathrm{~km}$ au large et loin des zones d'intérêt à $25 \mathrm{~m}$ au niveau des littoraux de Charente maritime et de Vendée. La résolution à la côte a été dictée par la nécessité de représenter convenablement les zones de déferlement pendant Xynthia. Le pas de temps hydrodynamique a été fixé à $60 \mathrm{~s}$ pour les deux modèles après avoir réalisé des tests de sensibilité et les simulations ont été démarrées au 10/02/2010.

\section{Résultats}

\subsection{Prévision des vagues}

Les prévisions de notre système de modélisation en termes de vagues ont été comparées avec les mesures disponibles dans le Golfe de Gascogne pendant Xynthia. La figure 3 


\section{XII ${ }^{\text {èmes }}$ Journées Nationales Génie Côtier - Génie Civil \\ Cherbourg, 12-14 juin 2012}

montre une comparaison avec les mesures réalisées par le Service Hydrographiques et Océanographique de la Marine au large de l'Île d'Oléron (figure 1). Cette comparaison montre que les $\mathrm{Hs}, \mathrm{Tp}$ et Dir sont reproduites avec des erreurs quadratiques moyennes de l'ordre de $0.5 \mathrm{~m}, 2.8 \mathrm{~s}$ et $24^{\circ}$, respectivement. Pendant Xynthia, le ratio entre des Hs de l'ordre de 4 à $7 \mathrm{~m}$ et des $\mathrm{Tp}$ de l'ordre de 7 à $10 \mathrm{~s}$ indique un état de mer très jeune avec des vagues très cambrées. Dans le détail, notre modèle sous-estime les chutes de Tp et Dir observées au début de la tempête (figure 3).
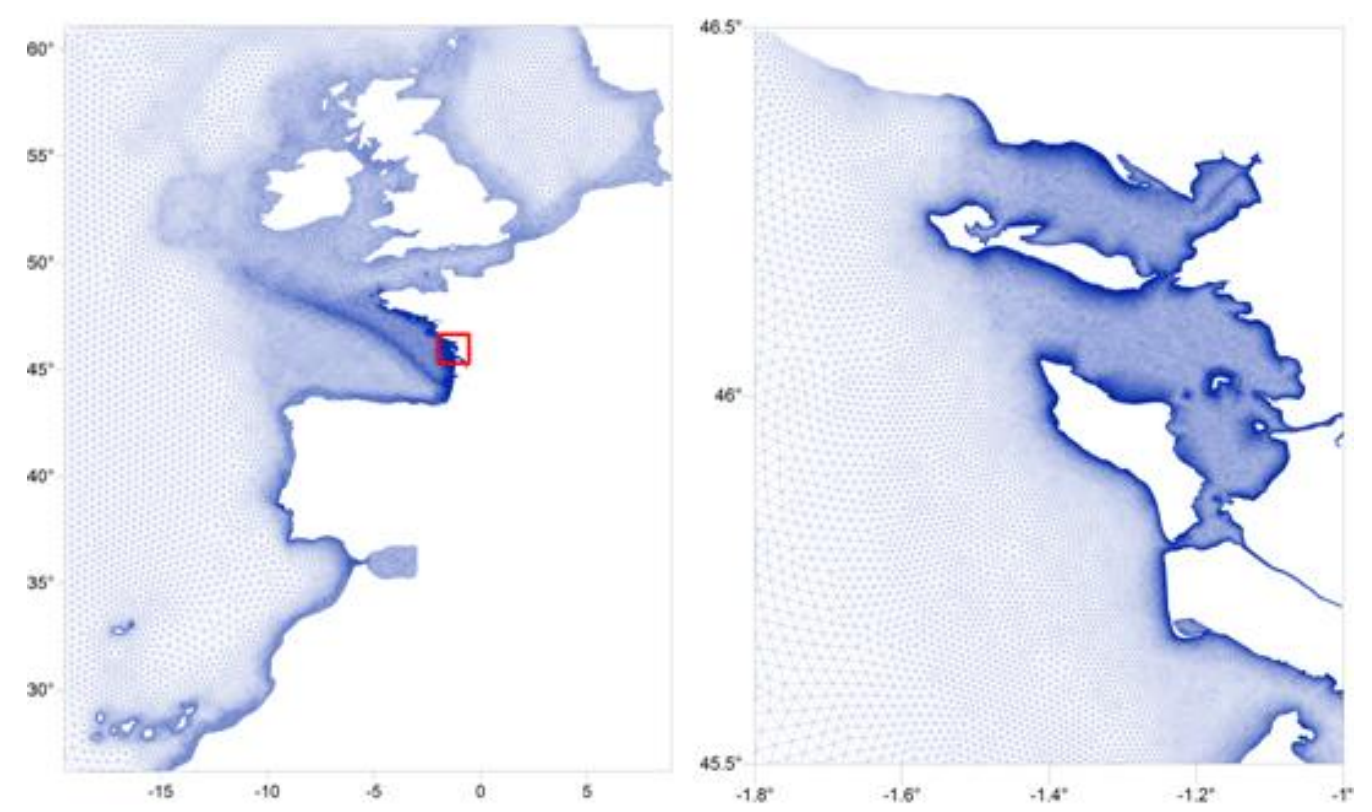

Figure 2. Grille de calcul non-structurée dont la résolution spatiale varie de $30 \mathrm{~km}$ au large à $25 \mathrm{~m}$ au niveau des littoraux de Charente-Maritime.
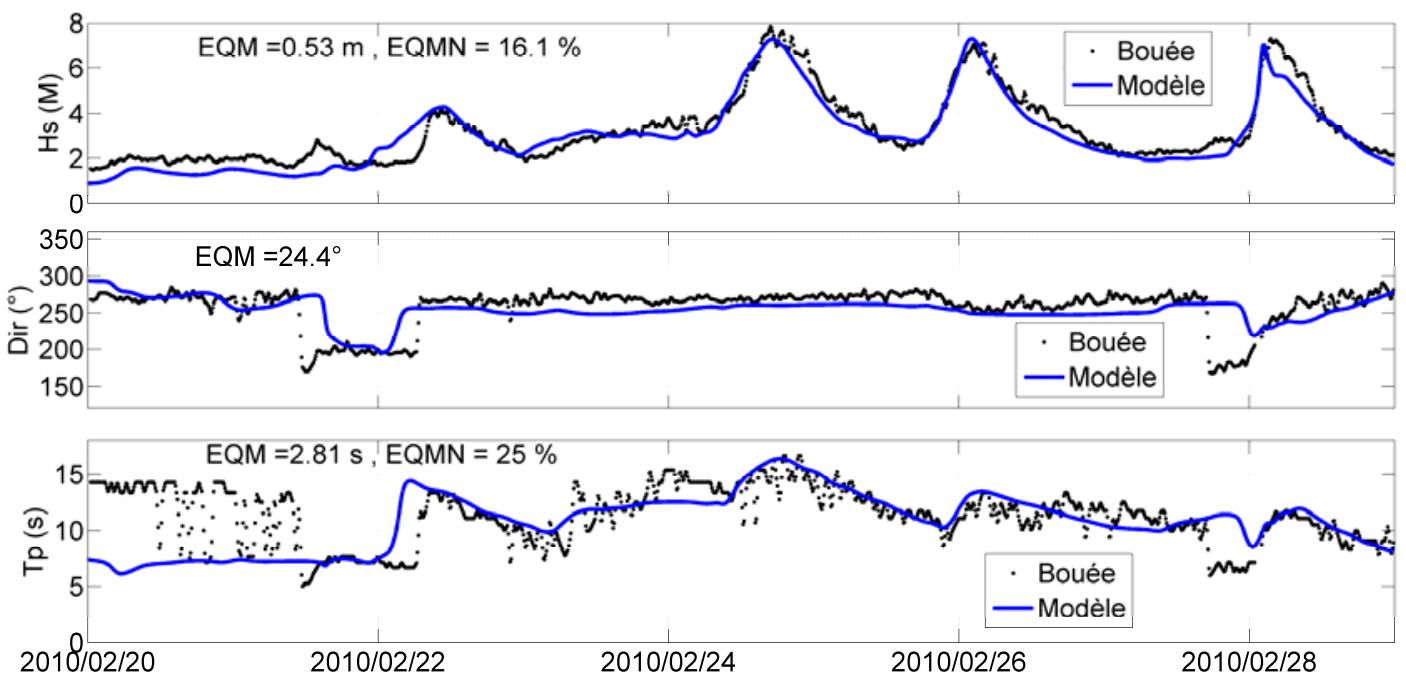

Figure 3. Comparaison entre les prévisions du modèle et les principaux paramètres de vagues mesurés au large de l'Île d'Oléron (figure 1). 


\subsection{Prévisions des surcotes}

Les prévisions de notre système de modélisation en termes de surcote ont été comparées avec la surcote déduite des mesures du marégraphe de La Pallice (LPA, figure 1). Cette comparaison montre que l'utilisation d'une paramétrisation quadratique pour calculer la contrainte liée au vent sous-estime la surcote d'environ $0.35 \mathrm{~m}$ (figure $4 \mathrm{~A}$, courbe magenta). L'utilisation d'une paramétrisation dépendante de vagues permet de reproduire la surcote de façon bien plus réaliste, avec légère une sous-estimation de l'ordre de 0.05 à $0.10 \mathrm{~m}$ (figure 4-A).
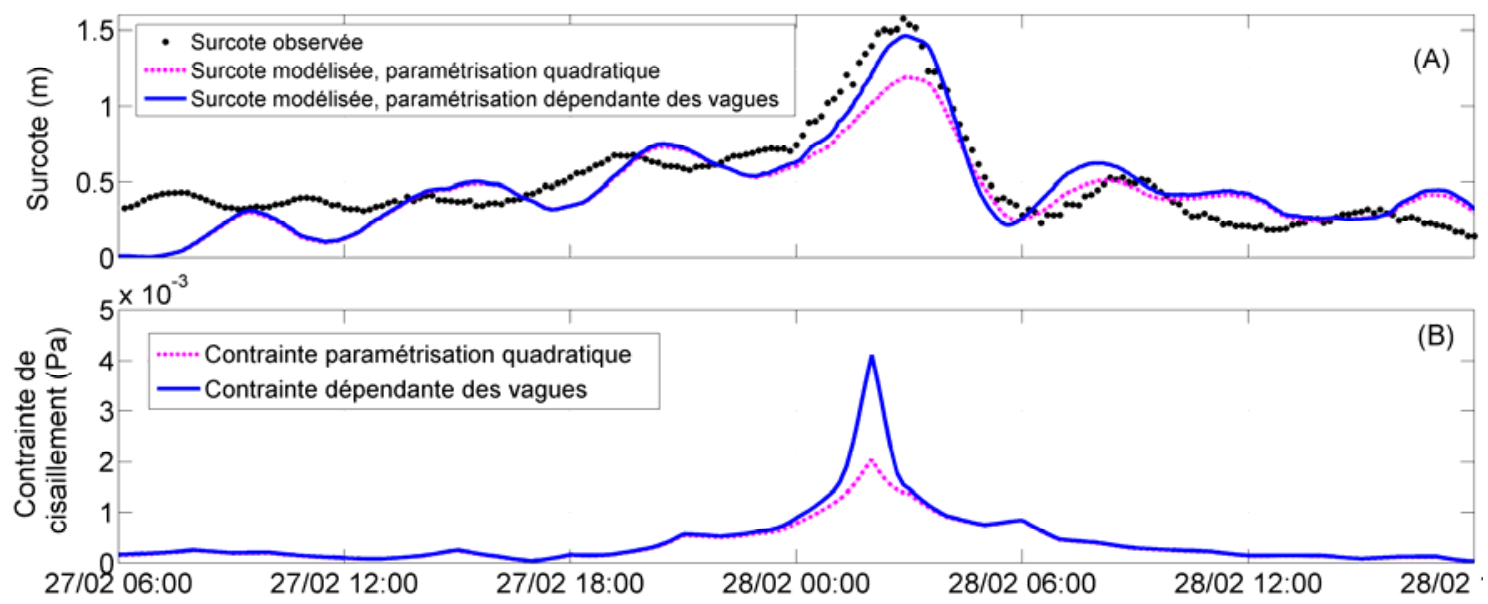

Figure 4. (A) Surcote observée (noirs), surcote modélisée avec une paramétrisation quadratique (magenta) et dépendant des vagues (bleue); (B) contrainte de cisaillement exercée par le vent pour les deux approches au niveau de la bouée "Oléron ».

\section{Discussion}

\subsection{Importance de la rugosité de l'océan en présence de vagues}

La comparaison entre la surcote prédite avec une paramétrisation quadratique et une paramétrisation dépendante des vagues montre que la seconde permet des prévisions bien plus réalistes, avec une différence de l'ordre de $20 \%$ entre les deux approches à la Pallice (figure 4-A). L'amélioration des prévisions de surcote en prenant en compte les vagues a déjà été reportée dans la littérature (e.g. MASTENBROAK et al., 1993) et est liée à un état de mer particulier pendant Xynthia. Les mesures comme le modèle ont montré la présence de vagues très courtes et cambrées pendant Xynthia (figure 3). Cet état de mer augmente fortement la rugosité de l'océan et donc la contrainte liée au vent (figure 4-B) et explique la prévision d'une surcote plus importante en prenant en compte les vagues. Cet état de mer particulier peut être expliqué par la trajectoire atypique de Xynthia du sud-ouest vers le nord-est du Golfe de Gascogne (figure 1), qui réduit la zone de génération des vagues à quelques centaines de $\mathrm{km}$ seulement. 


\section{XII ${ }^{\text {èmes }}$ Journées Nationales Génie Côtier - Génie Civil \\ Cherbourg, 12-14 juin 2012}

\subsection{Importance des vagues dans les zones de déferlement}

La figure 5 montre une répartition spatiale du maximum de la surcote calculée en prenant en compte les gradients de tension de radiation des vagues (A) et en ne les prenant pas en compte (B). Cette figure montre que la différence atteint localement 0.4 $\mathrm{m}$ le long de la cote ouest de l'Île d'Oléron (figure 5-C), ce qui correspond au setup induit par les vagues. De façon plus surprenante, ce mécanisme augmente également la surcote de l'ordre de $0.05 \mathrm{~m}$ dans les pertuis Charentais pour des profondeurs pour lesquelles il n'y a pas de déferlement bathymétrique. Ce comportement est expliqué par la présence de zones de déferlement kilométriques autour des îles de Ré et d'Oléron, qui induisent une surélévation du plan d'eau à l'échelle des Pertuis Charentais.
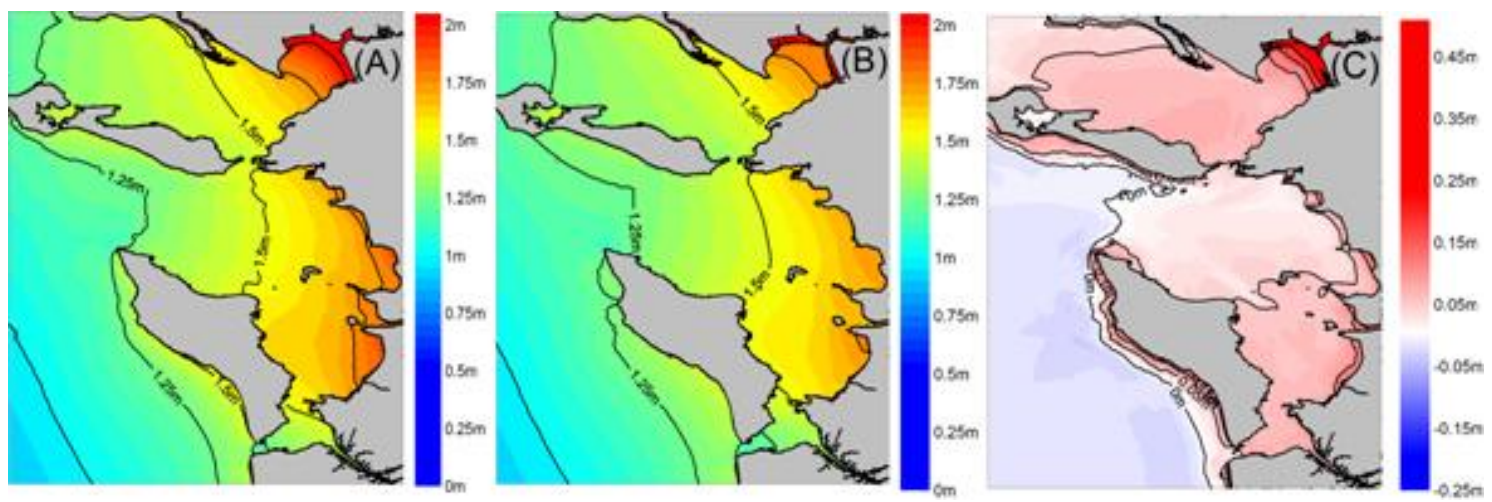

Figure 5. Carte d'iso-valeurs du maximum de la surcote calculée avec les gradients de tension de radiation des vagues $(A)$, sans $(B)$ et différence entre les deux approches $(C)$.

\section{Conclusions}

Notre système de modélisation numérique permet de reproduire les vagues et les surcotes dans les pertuis Charentais avec une bonne précision pendant Xynthia. L'analyse des résultats du modèle montre tout d'abord une forte amplification de la surcote du fait de la présence de vagues jeunes et cambrées, qui augmentent la rugosité de l'océan. Cet état de mer particulier est lié à la trajectoire atypique de la tempête Xynthia. Le setup induit par les vagues atteint localement $0.4 \mathrm{~m}$ au niveau des littoraux directement exposés à la tempête, et, de façon surprenante, n'est pas négligeable dans les zones complètement abritées de l'agitation. Sachant que les niveaux d'eau sont désormais prédits de façon précise le long de la cote, les perspectives à court terme de notre équipe consistent à étendre notre grille de calcul vers les zones inondées pendant Xynthia, afin de réaliser des simulations dynamiques de cette inondation.

\section{Remerciements}

Les données de hauteur d'eau ont été obtenues grâce au système d'observation SONEL (www.sonel.org). Les auteurs remercient chaleureusement l'équipe développant de code 
Thème 7 - Océanographie opérationnelle et situations extrêmes

SELFE (CMOP, EUA). Les données de vague au large d'Oléron of été fournies par le SHOM et acquises dans le cadre des programmes MOUTON (DGA PEA 012401) et EPIGRAM (LEFE/IDAO etANR-08-BLAN-0330-01). Les forçages atmosphériques proviennent de Météo France. Le projet "submersion marine" de 1'UMR LIENSs bénéficie du soutien du FEDER, du CG17 et de la Région Poitou Charente.

\section{Références bibliographiques}

ABGRALL R. (2006). Residual distribution schemes: Current status and future trends. Computers and Fluids 35, pp 641-669. doi:10.1016/j.compfluid.2005.01.007

BERNARDARA P., ANDREEWSKY M., BENOIT M. (2011). Application of regional frequency analysis to the estimation of extreme storm surges. Journal of Geophysical Research 116, C02008. doi:10.1029/2010JC006229

BERTIN X., BRUNEAU N., BREILH J.F., FORTUNATO A.B., KARPYTCHEV M., (2012). Importance of wave age and resonance in storm surges: the case Xynthia, Bay of Biscay. Ocean Modelling 42 (4), pp 16-30. doi:10.1016/j.ocemod.2011.11.001

DODET G., BERTIN X., TABORDA R., (2010). Wave climate variability in the NorthEast Atlantic Ocean over the last six decades. Ocean Modelling 31, pp 120-131. doi:10.1016/j.ocemod.2009.10.010

DUPUIS H., MICHEL D., SOTTOLICHIO A., (2006). Wave climate evolution in the Bay of Biscay over two decades. Journal of Marine Systems 63, pp 105-114. doi:10.1016/j.jmarsys.2006.05.009

MASTENBROEK C., BURGERS G., JANSSEN P.A.E.M. (1993). The dynamical coupling of a wave model and a storm surge model through the Atmospheric Boundary Layer. Journal of physical Oceanography 23, pp 1856-1866. doi:10.1175/15200485(1993)023<1856:TDCOAW >2.0.CO;2

PAIRAUD I.L., LYARD F., AUCLAIR F., LETELLIER T., MARSALEIX P. (2008). Dynamics of the semi-diurnal and quarter-diurnal internal tides in the Bay of Biscay. Part 1: Barotropic tides. Continental shelf Research 28, pp 1294-1315. doi:10.1016/j.csr.2008.03.004

ROLAND A., CUCCO A., FERRARIN C., HSU T.-W., LIAU J.-M., OU S.-H., (...), ZANKE U. (2009). On the development and verification of a 2-D coupled wave-current model on unstructured meshes. Journal of Marine Systems 78, pp 244-254. doi:10.1016/j.jmarsys.2009.01.026

ZHANG Y.L., BAPTISTA A.M. (2008). SELFE: a semi-implicit Eulerian-Lagrangian finite element model for cross-scale ocean circulation. Ocean Modelling 21, pp 71-96. doi:10.1016/j.ocemod.2007.11.005 